\title{
Devnagari Handwriting Recognition using STANN
}

\author{
Richa Sharma \\ M.Tech(CS) \\ Banasthali University \\ Rajasthan, India
}

\author{
Saurav Chandra \\ Associate Professor \\ CSE Department \\ KIET, Ghaziabad, India
}

\author{
Sanjeev Kumar Yadav \\ Associate Professor \\ CSE Department \\ KIET, Ghaziabad, India
}

\begin{abstract}
Several approaches to recognize the handwritten characters and numerals such as online cursive handwriting and numerals recognition have been proposed. Most of them are based on neural network approaches. In this paper, a technique for continuous recognition of handwritten Devanagari characters is proposed. This approach employs a method based on Spatio-Temporal Artificial Neural Network (STANN). The proposed method is efficient in the field of online handwriting recognition because of the property of STANN to detection of spikes of the continuous input signals. The method is based on different steps of recognition. The results of signals are extracted from the input handwritten characters where the spikes signals are generated by the continuous signals of the input character. A new algorithm using STANN is proposed with results in this paper.
\end{abstract}

\section{General Terms}

Devnagari handwriting recognition, online character recognition

\section{Keywords}

STANN (Spatio-Temporal Artificial Neural Network), Preprocessing, Spiking Neural Network, Spike Detection.

\section{INTRODUCTION}

In the field of handwriting recognition many researcher had been proposed their work. In past several years different types of neural networks have been proposed and among these neural networks most of them have most promising results in the fields of recognitions like pattern recognition, handwriting recognition. The brief overview on these networks can be found in [1]. Work on Devanagari handwriting recognition attracted to most of researchers doing their research, as Devanagari are the script which is used to write most of Indian languages. One of the techniques which can be applied to recognize Devanagari handwriting is STANN (SpatioTemporal Artificial Neural Network). The STANN is a new model of 3rd generation. Hodgkin and Huxley conductance based neuron model is becomes the father of spiking neurons. STANN is a type of Spiking Neural Network (SNN) [2]. Spiking neurons are powerful computing elements. These neurons use the timing of signals action potential or spikes which use to encode the information. The spiking neuron model is innately embedded in the time and these time and spatial information of spiking neurons gain fast computational power. Based on these discussions, an efficient method for recognizing Devanagari handwriting using STANN is proposed. In this paper, the proposed method is applied to recognize Devanagari handwriting. Due to Variations in Handwriting Styles, Personal or Background Factors, Situational Factors, Material Factors, Constrained and Unconstrained Handwriting, Limited Resources in Small Devices, Similarity in Shape of Some Characters and other different type of challenges recognition became difficult. During past years, several approaches have been proposed to overcome with these challenges [3].This paper is organized into five sections. In section 2, gives the overview of survey which has been done to propose this method. In Section 3, a brief introduction about the technology STANN and the method of preprocessing $[4,20]$ and generating signals using MATLAB. The proposed algorithm of recognizing Devanagari handwriting is presented in section 4. In section 5, the results and future scope of work is discussed. In section 6, we use a few concluding remarks to conclude the paper.

\section{LITERATURE SURVEY}

In several years different researchers proposed their work in different field of recognition. This section presents some of the selected recent results into the Table1 from literature of handwriting recognition systems. Most of the results given in this subsection are based on handwritten character recognition as present study is focused to this area only.

Different types of research have been done to recognize the Hindi numerals [5, 6]. In [1] the table shows the comparison between applications of different neural network models in the field of recognition. The comparison of different neural networks in recognition fields are shown in table 1 in this paper, which is as follows: 
Table 1. A Survey on Proposed Work on Handwriting Recognition

\begin{tabular}{|c|c|c|}
\hline Author(s) & Method Of Recognition & Year \\
\hline $\begin{array}{c}\text { Ashutosh Malaviya, } \\
\text { Hartmut Surmann } \\
\text { and Liliane peters } \\
\text { [7] }\end{array}$ & $\begin{array}{c}\text { Fuzzy Rule Base For } \\
\text { Online Handwriting } \\
\text { Recognition }\end{array}$ & 1994 \\
\hline $\begin{array}{l}\text { H.Bunke, M.Roth, } \\
\text { E.G.Schukat, } \\
\text { Talamazzini [8] }\end{array}$ & $\begin{array}{l}\text { Hidden Markov Model } \\
\text { For Off-line Recognition } \\
\text { of Cursive Handwriting }\end{array}$ & 1998 \\
\hline $\begin{array}{c}\text { Kam-Fai Chan, Dit- } \\
\text { Yan Yeung [9] }\end{array}$ & $\begin{array}{c}\text { Flexible Structural } \\
\text { Matching For on-line } \\
\text { Handwritten } \\
\text { Alphanumeric Characters }\end{array}$ & 1999 \\
\hline $\begin{array}{c}\text { S.D. Connell, R. M. } \\
\text { K. Sinha, A. K. Jain } \\
{[10]}\end{array}$ & $\begin{array}{l}\text { statistical method HMM } \\
\text { with neural network For } \\
\text { Unconstrained Online } \\
\text { Devnagari Characters }\end{array}$ & 2000 \\
\hline $\begin{array}{c}\text { Tay, Yong Haur } \\
{[11]}\end{array}$ & $\begin{array}{c}\text { Artificial Neural Network } \\
\text { (ANN) and Hidden } \\
\text { Markov Model (HMM) } \\
\text { For Offline Handwriting } \\
\text { Recognition }\end{array}$ & 2002 \\
\hline $\begin{array}{c}\text { Abdul Rahim hmad, } \\
\text { Christian Viard- } \\
\text { Gaudin, Marzuki } \\
\text { Khalid, Rubiyah } \\
\text { Yusof[12] }\end{array}$ & $\begin{array}{l}\text { Support Vector Machine } \\
\text { (SVM) For Online } \\
\text { Handwriting Recognition }\end{array}$ & 2004 \\
\hline A. Rauf Baig [13] & $\begin{array}{c}\text { Spatio-Temporal Artificial } \\
\text { Neuron (STAN) for } \\
\text { Online Cursive } \\
\text { Handwritten Character } \\
\text { Recognition }\end{array}$ & 2004 \\
\hline $\begin{array}{c}\text { Shashank Mathur, } \\
\text { Vaibhav Aggarwal, } \\
\text { Himanshu Joshi, } \\
\text { Anil Ahlawat [14] }\end{array}$ & $\begin{array}{c}\text { Artificial Neural Networks } \\
\text { and Genetic Algorithm for } \\
\text { Offline Handwriting } \\
\text { Recognition }\end{array}$ & 2008 \\
\hline $\begin{array}{c}\text { A.AZaidan, B.BZaid } \\
\text { an, Hamid A. Jalab } \\
\text {, Hamdan.O.Alanazi } \\
\text {, Rami Alnaqeib } \\
\text { [15] }\end{array}$ & $\begin{array}{l}\text { Artificial Neural Network } \\
\text { For Offline Arabic } \\
\text { Handwriting Recognition }\end{array}$ & 2010 \\
\hline $\begin{array}{c}\text { Deepika Wadhwa, } \\
\text { Karun } \\
\text { Verma[4] } \\
\end{array}$ & $\begin{array}{c}\text { Support Vector Machine } \\
\text { For Online Handwritten } \\
\text { Devanagari Numerals }\end{array}$ & 2012 \\
\hline $\begin{array}{c}\text { Bhushan C. Bhokse, } \\
\text { Bhushan S.Thakare } \\
{[16]}\end{array}$ & $\begin{array}{l}\text { Dynamic Time Warping } \\
\text { Algorithm for Devnagari } \\
\text { Handwriting Recognition }\end{array}$ & 2012 \\
\hline
\end{tabular}

\section{STANN (SPATIO-TEMPORAL} ARTIFICIAL NEURAL NETWORK)

STANN stands for "Spatio-Temporal Artificial Neural Network". Spatio-Temporal Artificial Neuron is an artificial neuron which codes discrete events using complex number. [17].The representation of Different types of input is illustrated in fig.1. Spatio-Temporal Artificial Neural Network is basically generates sequences of impulses on the basis of input signals. The mathematical description of SpatioTemporal Artificial Neural Network is as follows:

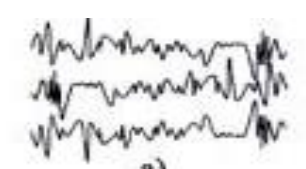

a)

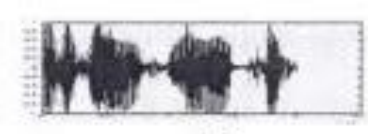

b)

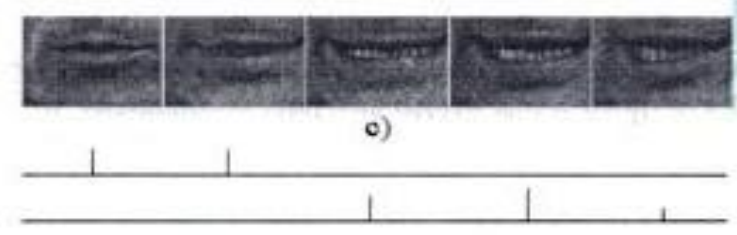

d)

Fig. 1. Different Input Signal: a) Raw input signal b) Continuous Signals c) Sequence of image d) impulse signals Ref. [17]

An event is represented as an impulse $z$; the spatial and temporal aspects of $\mathrm{z}$ are simultaneously taken into account by coding it in the complex domain [13]. In polar coordinates $(\partial, \theta)$ the magnitude $\partial$ gives the amplitude and the angle $\theta$ gives the temporal position (or age) of the impulse from a reference point.

$$
\begin{aligned}
& z=\delta e^{i \theta} \text { where } \tan (\theta)=\mu_{T} \tau \\
& \text { Hence, } \quad z=\partial e^{i \arctan \left(\omega_{T} \tau\right)}
\end{aligned}
$$

When a new impulse is emitted on a given component, it is gathered together with the previous impulses. The amplitude is made to decrease with time. Hence any given event is forgotten in due course of time. The impulse of amplitude emitted at time $\mathrm{t} 1$ is at current time $\mathrm{t}$ by the complex number:

$$
Z(t)=\delta e^{-\mu_{g} \tau} e^{-i \arctan \left(\mu_{T} \tau\right)}
$$

Where $\mu_{g}=\mu_{T}=1 / T W$, TW is stands for Temporal Window.

TW depends on the application and represents the size of the temporal window inside which one wishes to identify sequence of impulses. When each of impulse arrives and compared with stored vector W, Output of an input and W are close enough is illustrated in figure 2. [13].The STANs are spiking neurons. The weight $\mathrm{W}$ of a STAN is a complex vector and it represents the sequence to be detected by it. The 
comparison between $\mathrm{X}$ and $\mathrm{W}$ can be done by means of a Hermitien product $\mathrm{V}$ in one type of STAN and in another type it can be done by Hermitien distance D:

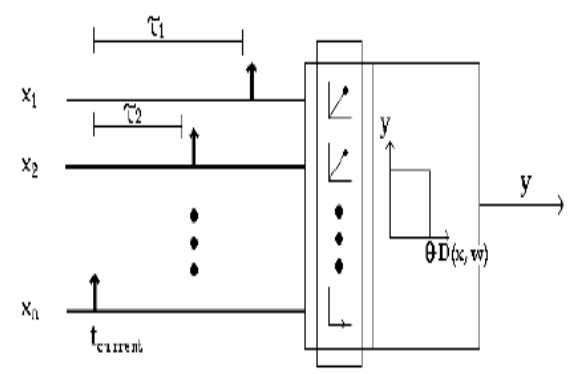

Figure 2: Arrival of impulses and comparison of stored vector $W$ with the output of input $X$ [11].

$$
\begin{gathered}
V(\mathrm{X}, \mathrm{W})=\sum_{\mathrm{j}=1}^{\mathrm{N}} \overline{\mathrm{W}_{0}} \mathrm{x}_{\mathrm{j}} \\
\text { And } \\
D\left(X_{,} W\right)=\sqrt{\sum_{j=1}^{W}\left(x_{j}-w_{j}\right) \overline{\left(x_{p}-w_{j}\right)}}
\end{gathered}
$$

The bar denotes the complex conjugate. In figure 2, its shows how the value of $\mathrm{D}$ is calculated corresponding to the input value.

\section{RECOGNITION METHOD}

To recognize the character using STANN is according to the following module: (1) First module is the data inputting and preprocessing phrase $[2,20]$ which will be done using different types of algorithm in various steps of preprocessing. (2)Second module is the combination of feature extraction of the character and the generation of continuous signals for the further steps of the proposed work. It will done using the MATLAB software and the wavelet transformation [18] to extracting the impulse signals for recognition of character input. (3) Third Module is the compression of signals to perform the recognition. Recognition is done by comparing the impulse signals to each other and the compression by which best matching is deemed to be recognized character. The proposed algorithm of recognizing Devanagari handwriting involves these steps as follows:

Step1. Input the character using mouse on the writing area of $300 * 300$ pixels given on the screen. In this area the character can be written in any size or shape. The next step is to normalize the characters which can be performed by step 2 .

Step2. In this step of normalization the size of written character is fixed into a constant frame of size:

begin ( $\mathrm{k}$ is any positive integer)

for $\mathrm{k}$ from 1 to appwindow size begin

Set k of xnew to ((195.0 / (appwindow. maxX - appwindow. $\min X))$ )
Set k of xnew to position $\mathrm{k}$ in xnew +300 ;

Set k of ynew to ((195.0/ (appwindow. maxY- appwindow. $\min Y))$ )

Set $\mathrm{k}$ of ynew to position $\mathrm{k}$ in ynew plus 100 end;

end;

Step3. Find the distance between two consecutive points using following method:

begin ( $\mathrm{j}$ is any positive integer)

for $\mathrm{j}$ from 1 to appwindow.i-2

Set $x$ to Math.abs with (position in xnew - position $\mathrm{j}$ in xnew)

Set $\mathrm{j}$ of dis to Math. sqrt with $\mathrm{x}$ end;

if $\operatorname{dis}[\mathrm{j}]>1$

missing point interpolation method is applied else

go to step 4.

Step4. Resample the points by initializing the resampled point to "64".

String str = "64";

int option;

If (str! = null)

Set appwindow.len1 to str else

Set option to CLOSED_OPTION end

Step5. Smoothing process is done by considering two neighbor of each side of the point if three points are considered then nature of the stroke may be lost.

Step6. Due to different handwriting styles problem of slant and bend is occurred. To overcome the problem eight directional chain code methods [18] is applied to the input character. After applying this method the extracted features are stored into the .csv file.

Step7. Signals of the input character are generated by using MATLAB wavelet toolbox. A result shows in figure 3-4.

Step8. After detecting the spike signals [19] of character in step7, the character recognition process is performed by signal compression using MATLAB software.

\section{RESULT AND FUTURE WORK}

To evaluate the working of the proposed method of recognizing Devanagari handwriting the method is applied on different Devanagari characters. In figure 3, the results of input the character in writing area is shown and from figure 45 , the results of generated signals and the detection of spikes of continuous signals is shown. In this proposed methods we just select some of the samples. The evolution of recognition method on different samples will be performed in future. The recognition method has works well with the recognition of Hindi numerals and some of the character of Devanagari, but it needs to be more efficient with other Devanagari characters which have similarity in nature and written with difficult strokes. The present work can be extended for recognition of the other handwritten characters. 


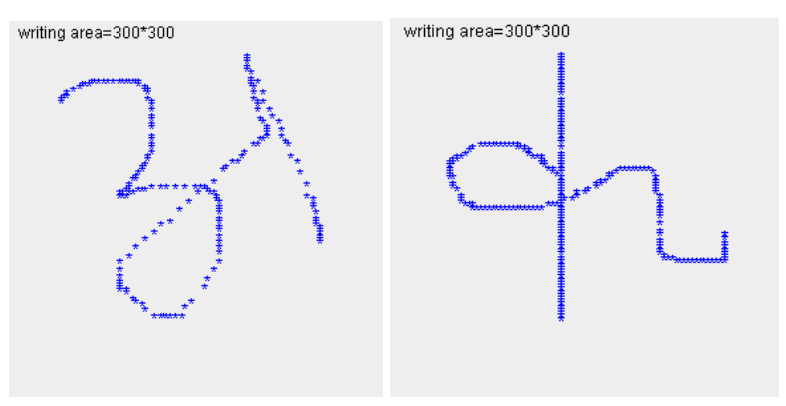

Fig. 2. Input character $अ$ and $क$ using the mouse on the $300 * 300$ pixel writing area
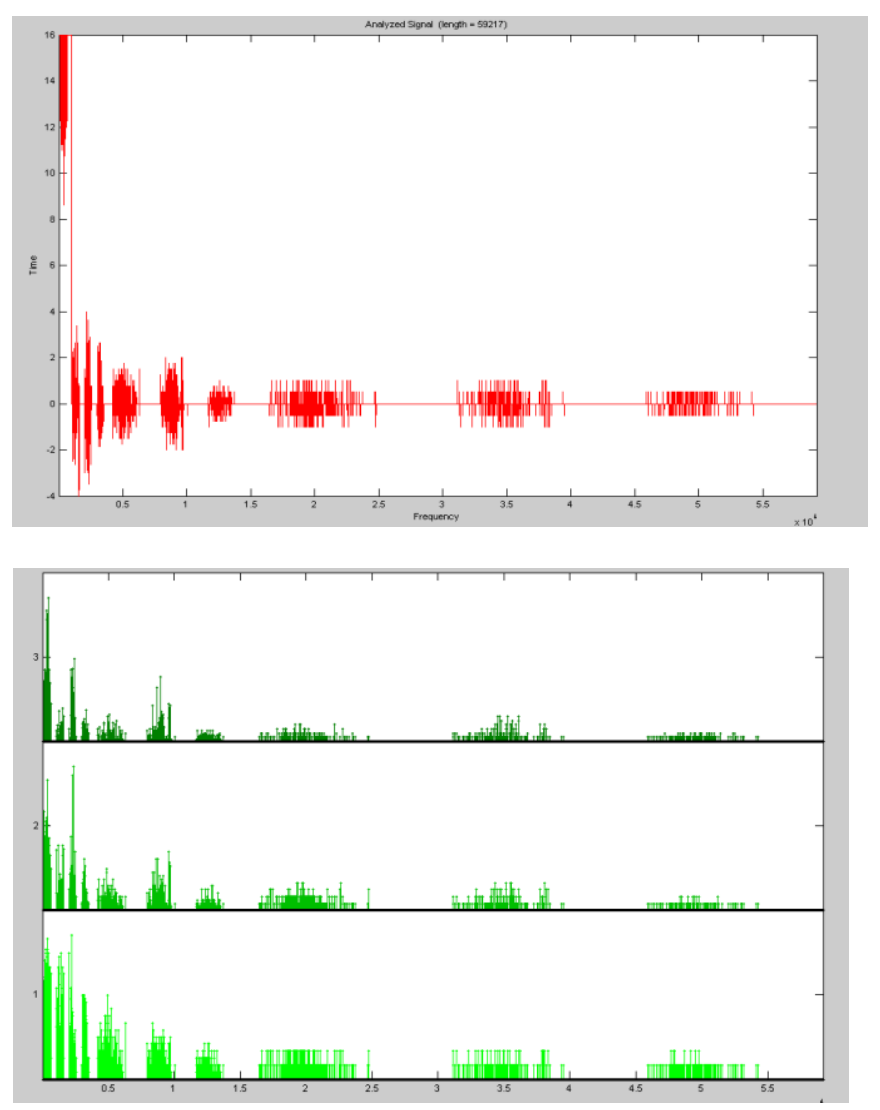

Fig. 3. Generated signal and spike detection of character "क " by MATLAB with the help of stored co-ordinates

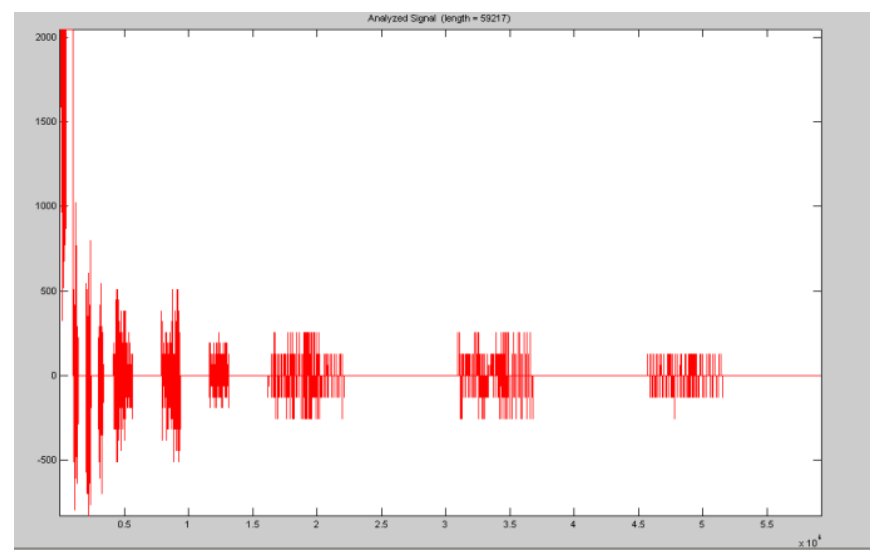

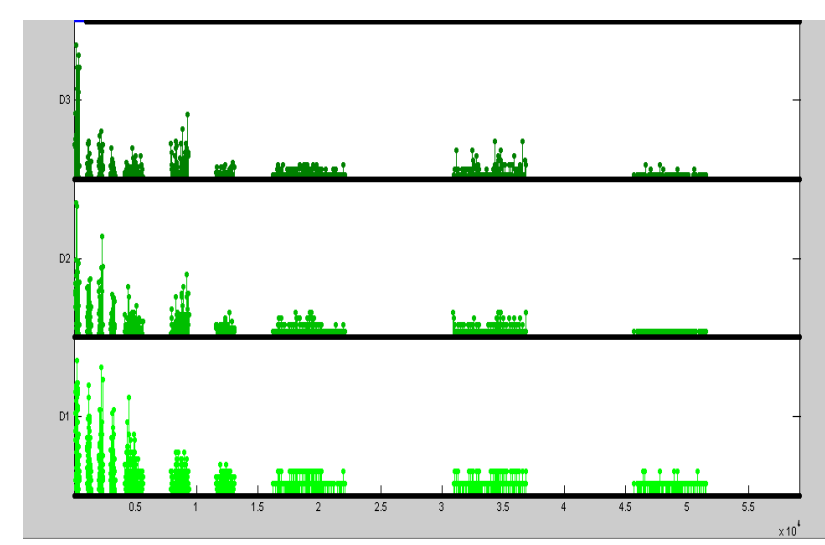

Fig. 4. S Generated signal and pikes detection of signals of character " अ" by MATLAB with the help of stored coordinates

\section{CONCLUSION}

A method of recognizing Devanagari recognition is proposed in this paper. The signals of the Devanagari character can be generated efficiently and the spikes detection of signals can be performed effectively and the comparison of these signals done for some characters but will be done for other characters in future. The work can be extended by using different type of other input and preprocessing methods for applying STANN technology. In this paper an algorithm for recognizing Devanagari handwriting is proposed.

\section{ACKNOWLEDGMENTS}

I would like to express my special thanks to my friend Ms. Deepika Wadhwa, for her kind support and help me to work on this area because she had also worked on the same area. She always becomes milestone on the way of my work as a good friend.

\section{REFERENCES}

[1] Sharma Richa, 2013. Application of Neural Network Models in Recognition Field: A Survey. International Journal of Scientific \& Engineering Research, Vol.4-2.

[2] Maass, Wolfgang, 1997. Networks of spiking neurons: the third generation of neural network models. Elsevier, Neural Networks Vol. 10-9.

[3] Tappert, C. C., 1984. Adaptive Online Handwriting Recognition. Proceedings of International Conference of Pattern Recognition, pp. 1004-1007.

[4] Wadhwa Deepika, Verma Karun, 2012. Online Handwriting Recognition of Hindi Numerals using SVM. International Journal of Computer Applications, Vol. 4811.

[5] Dr. Mahdy, Y. B, ENG El-Melegy, 1996. Encoding Patterns for Efficient Classification by Nearest Neighbor Classifiers and Neural Networks with Application to Handwritten Hindi Numeral Recognition. Proceedings of ICSP, M. T.

[6] Hanmandlu, M., Grover, J. Madasu, V.K., and Vasikarla, S., 2007. Input Fuzzy Modeling for the Recognition of Handwritten Hindi Numerals. In: International Conference on Information Technology. 
[7] Malaviya Ashutosh, Surmann Hartmut and peters Liliane, 1994. Automatic Generation of a Fuzzy Rule Base for Online Handwriting Recognition. Second European Congress on Intelligent Techniques and Soft Computing (EUFIT), Aachen, pp. 1060-1065.

[8] Bunke H., Roth M., E. G. Schukat Talamazzini, 1995. Off-line Recognition of Cursive Handwriting Based on Hidden Markov Models (HMMs). Pattern Recognition, Vol.28- 9.

[9] Kam-FaiChan, Dit-YanYeung, 1999. Recognizing Online Handwritten Alphanumeric Characters through Flexible Structural Matching. Pattern Recognition-32.

[10] S. D. Connell, R. M. K. Sinha, A. K. Jain, 2000. Recognition of Unconstrained Online Devanagari Characters. ICPR.

[11] Tay, Yong Haur, 2002. Offline Handwriting Recognition using Artificial Neural Network and Hidden Markov Model. Thesis (Doctor of Philosophy)Universities Technology Malaysia.

[12] Abdul Rahim hmad, Christian Viard-Gaudin, Marzuki Khalid, Rubiyah Yusof, 2004. Online Handwriting Recognition using Support Vector Machine. Second International Conference on Artificial Intelligence in Engineering \& Technology.

[13] A. Rauf Baig, 2004. Spatial-Temporal Artificial Neurons Applied to Online Cursive Handwritten Character Recognition. European Symposium on Artificial Neural Networks, Bruges (Belgium).
[14] Shashank Mathur, Vaibhav Aggarwal, Himanshu Joshi, Anil Ahlawat, 2008. Offline Handwriting Recognition using Genetic Algorithm. Sixth International Conference on Information Research and Applications-i.Tech, Varna, Bulgaria.

[15] A.AZaidan, B.B.Zaidan, HamidA.Jalab, Hamdan.O.Alan azi, Rami Alnaqeib, 2010. Offline Arabic Handwriting Recognition Using ANN. Journal of Computer Science and Engineering, Vol.1, pp. 55-58.

[16] Bhushan C. Bhokse, Bhushan S.Thakare, 2012. Devnagari Handwriting Recognition System using Dynamic Time Warping Algorithm. International Journal of Computer Applications (0975 - 8887) Vol. 52-9.

[17] Sivanandam S., Deepa S. N., Sumathi S, 2006. Introduction to Neural Networks Using MATLAB 6.0.Tata McGraw-Hill Education.

[18] Ding Y., Kimura F. and Miyake Y., 2004. Slant Estimation for Handwritten Words by Directionally Refined Chain Code. University of Groningen Archive, pp. 1-10.

[19] Nenadic Zoran, Joel W. Burdick, 2005. Spike Detection using the Continuous Wavelet Transform. In: Biomedical Engineering, IEEE Transactions Vol.52-1, pp. 74-87.

[20] Wadhwa Deepika, 2012. Recognition of Online Handwritten Devanagari Numerals using Support Vector Machine. M.Tech. Thesis, Thapar University, June. 